directional sequencing (SBS) has been used as the gold standard for EGFR mutational analysis, however there are emerging new assays utilizing targeted real time PCR technology. In this study we compared the performance of 'Cobas 4800' (COBAS) against Sanger sequencing.

Methods: 480 consecutive formalin fixed paraffin embedded samples of lung adenocarcinoma were simultaneously tested for EGFR mutations by SBS and COBAS. Mutational results were catogorised as positive, negative and invalid. Unweighted Kappa test was utilsed to compare the concordance between two assays. Results: After exclusion of invalid results $(n=16), 477$ samples from 458 patients $(47.2 \%$ male, $52.8 \%$ female) underwent statistical analysis. There was an excellent observed percentage agreement of $98.3 \%$ (kappa value 0.944 , SE 0.0194) between the two methods. The combined mutation detection rate (19\%) was superior to either SBS $(18.4 \%)$ or COBAS $(18 \%)$. EGFR mutation frequency was significantly higher in women $(23 \%)$ compare to men (12\%).

Discussion: COBAS assay is a diagnostically robust platform comparable with Sanger with very high analytical sensitivity and short turns around time. COBAS failure is due to lower sensitivity to samples with low DNA quality and its limited primer detection range, while Sanger is mostly affected by its lower analytic sensitivity related to low volume of diagnostic material. Consequently, the higher combined mutation detection rate necessitates a dual testing strategy to guarantee detection of novel mutations and mutations outside the COBAS detection range, and avoiding false negative results due to lower analytical sensitivity of SBS.

\section{MOLECULAR PROFILING STUDY OF A RECURRENT OVARIAN MUCINOUS TUMOUR WITH A MURAL NODULE OF ANAPLASTIC CARCINOMA, PROVIDING SUPPORTIVE EVIDENCE OF DEDIFFERENTIATION}

Nima Mesbah Ardakani ${ }^{1}$, Anup Naran ${ }^{2}$, Fabienne Grieu-Iacopetta ${ }^{1}$, Colin Stewart ${ }^{2}$ ${ }^{1}$ Anatomical Pathology Department, PathWest, QEII, and ${ }^{2}$ Anatomical Pathology Department, PathWest, KEMH, Perth, Australia

Background: Malignant mural nodule in ovarian mucinous tumours is an exceedingly rare occurrence. The mural nodules can be of sarcomatous or carcinomatous differentiation; however it is not clear if this is a result of dedifferentiation in the mucinous tumour or simply a collision phenomenon.

Clinical data: A 38-year-old woman presented with a $21 \mathrm{~cm}$ right ovarian cystic mass, which was resected surgically. The patient had a previous right ovarian cystectomy 7 years ago with a diagnosis of low-grade borderline mucinous tumour.

Pathological findings: Grossly there was a necrotic cystic mass weighting $547 \mathrm{~g}$ with thickened wall containing a focal mural nodule up to $2 \mathrm{~cm}$ in diameter. Microscopic examination revealed an infarcted cystic lesion with ghost outlines of multilayered polygonal cells and viable fibrous stroma with lakes of extruded mucin, which were overall interpreted as a recurrent unclassifiable mucinous neoplasm. The mural nodule was composed of viable anaplastic rhabdoid cells with pleomorphic nuclei, eosinophilic cytoplasm and occasional mitotic figures. Immunophenotypically the rhabdoid cells showed strong cytokeratin positivity in keeping with anaplastic carcinoma. The previous cystectomy specimen was reviewed which showed features of a borderline mucinous tumour.

Molecular analysis: Appropriate formalin fixed paraffin embedded sections of the anaplastic carcinoma and previously resected mucinous tumour were micro-dissected and DNA was extracted for next generation sequencing. Gene profiling showed a similar K-RAS mutation (c.35 G>T; p.Gly12Val) in both anaplastic carcinoma and previous borderline mucinous tumour. Discussion and conclusion: Finding of a similar K-RAS mutation in both viable anaplastic carcinoma and previously resected mucinous tumour is a supportive evidence for a recurrence, and that the nodule of anaplastic carcinoma likely to represent a dedifferentiation phenomenon in same clonal neoplastic cells rather than a collision tumour.

\section{EXPRESSION OF PROTEIN REGENERATING LIVER-3 (PRL-3) AND E-CADHERIN IN COLORECTAL CANCER}

Upik Anderiani Miskad, Muhammad Husni Cangara,

Syarifuddin Wahid

Department of Pathology, Faculty of Medicine, Hasanuddin

University, Makassar, Indonesia

Background: Colorectal cancer is the third most common malignant neoplasm worldwide. PRL-3 (phosphatase of regenerating liver-3/PTP4A3) was reported participates in the progression of colorectal cancer and play a role in epithelial to mesenchymal transition by down regulate the expression of E- cadherin.

Aims: To clarify the molecular mechanisms that involved in colorectal cancer development and progression, we investigate the expression of PRL-3 and E-Cadherin in colorectal cancer and correlate the expression with the clinicopathologic parameters. Methods: Expression of PRL-3 and E-Cadherin in 76 colorectal cancer specimens were detected by immunohistochemistry.

Results: Among colorectal cancer specimens examined, there were $30(39.5 \%)$ well differentiated, $36(47.4 \%)$ moderately differentiated and $10(13.2 \%)$ poorly differentiated CRC. There were significant correlation between histological grading and PRL-3 expression $(p=0.044)$, and also with E-cadherin expression $(p=0.039)$. The expression of PRL-3 was significantly correlated the expression of $\mathrm{E}$ cadherin in colorectal cancer $(p=0.003)$. The result showed that the more higher PRL-3 expression the more lower the expression of E-cadherin in colorectal cancer.

Conclusions: These studies strongly suggest that PRL-3 may play a role to down regulate the expression of $\mathrm{E}$ cadherin in the development and progression of colorectal cancer.

\section{COLORECTAL ADENOCARCINOMA WITH THE SERRATED NEOPLASIA PATHWAY}

Renn Montgomery, Tao Yang

Department of Anatomical Pathology, Liverpool Hospital, Liverpool, NSW, Australia

The conventional pathway for the development of colorectal carcinoma (CRC) has been well described, and involves mutations in the APC gene, as well as alterations in p53 and the development of microsatellite instability. A more recently described pathway implicates sessile serrated lesions in the 
development of colorectal adenocarcinoma, illustrated by a case we report of a 66 year old male presenting with three synchronous adenocarcinomas, with multiple sessile serrated adenomas (SSAs). BRAF V600E mutation was detected and there was sporadic microsatellite instability.

The serrated neoplasia pathway differs from the conventional pathway, involving mutations in the BRAF oncogene, as well as epigenetic mechanisms leading to abnormal hypermethylation of tumour suppressor genes. This methylation may involve MLH1, leading to sporadic microsatellite instability, as seen in our case. Sessile serrated lesions are the precursor for this pathway, and it is possible that they may progress to carcinoma more rapidly than conventional adenomas. They are often difficult to identify and completely remove. Thus, screening of SSAs and identification of SSA polyposis to prevent CRC may be of particular clinical significance.

\section{IMMUNOHISTOCHEMICAL EXPRESSION AND CLINICAL SIGNIFICANCE OF SUGGESTED STEM CELL MARKERS IN HEPATOCELLULAR CARCINOMA}

Jong Jin Sung $^{1}$, Jun Sang Bae ${ }^{1}$, Sik Lee ${ }^{2}$, Kyu Yun Jang ${ }^{1}$, Woo Sung Moon ${ }^{1}$

${ }^{1}$ Department of Pathology, and ${ }^{2}$ Internal Medicine, Chonbuk National University, Medical School, and Research Institute for Endocrine Sciences, Jeonju, Republic of Korea

Background: Accumulating evidence suggests that tumor maintenance and growth are sustained by a minority population of cancer stem cells (CSCs) or tumor-propagating cells. CSCs are posited to be responsible for tumor initiation and for the generation of distant metastasis and relapse after therapy.

Aims: We examined the expression of suggested CSC markers in paraffin-embedded tissue sections of hepatocellular carcinoma (HCC) and correlated with the clinicopathologic characteristics. Methods: Immunohistochemical staining of CSC markers: EpCAM, Keratin 19 (K19), CD133 and CD56 were performed in 82 HCC specimens.

Results: The expression of EpCAM was observed in 56\% (46/ 82) of the HCCs. K19 expression was observed in 5 of 82 samples $(6 \%)$. EpCAM expression in HCC was significantly associated with elevated alpha-fetoprotein level, vascular invasion, and high tumor grade. In addition, EpCAM expression was strongly associated with K19 expression. The 5-year survival rate in patients with EpCAM-positive HCC was lower than that of patients with EpCAM-negative HCC. All but two of the $82 \mathrm{HCCs}$ were negative for CD133 and CD56, respectively.

Conclusions: Our results suggest that HCCs expressing EpCAM are associated with unfavorable prognostic factors and have a more aggressive clinical course. Both CD133 and CD56 expression in paraffin-embedded HCC tissue is rare.

\section{EXTRACT ETHANOL OF CENTELLA ASIATICA REDUCE EXPRESSION OF MYCOBACTERIUM TUBERCULOSIS ANTIGEN ON ALVEOLAR MACROPHAGE FROM RATS LUNG TSSUE INFECTED WITH MYCOBACTERIUM TUBERCULOSIS}

Arifa Mustika, Mangestuti Agil, Sri agus Sudjarwo,

Ni Made Mertaniasih
Airlangga University, Indonesia

Centella asiatica is a medicinal plant that has been used as a traditional treatment for healing inflammatory processes. Previous studies have shown that the extract of Centella asiatica is able to increase the immune response and can inhibit the growth of Mycobacterium tuberculosis in vitro. The aims of this study were to determine the effect of ethanol extract of Centella asiatica to reduce the expression of antigen of Mycobacterium tuberculosis on alveolar macrophage cells from rat lung tissue infected with Mycobacterium tuberculosis.

Twenty-eight male rats were infected with Mycobacterium tuberculosis strain $\mathrm{H} 37 \mathrm{Rv}$ and divided randomly into four groups. Groups 1, 2, and 3 were treated with the ethanol extract of Centella asiatica at $375 \mathrm{mg} / \mathrm{kgBW}, 750 \mathrm{mg} / \mathrm{kgBW}$ and $1500 \mathrm{mg} / \mathrm{kgBW}$, and fourth group was the control group. Examination of Mycobacterium tuberculosis antigen expression using immunohistochemical methods was performed. Analysis of the data using ANOVA $(\alpha=0.05)$.

The results showed that there was a significant reduction of Mycobacterium tuberculosis antigen expression in alveolar macrophage in the treated group at $750 \mathrm{mg} / \mathrm{kgBW}$ and $1500 \mathrm{mg}$ / $\mathrm{kgBW}$.

In conclusion, the ethanol extract Centella asiatica contains active ingredients that have the ability to decrease the expression of Mycobacterium tuberculosis antigen on alveolar macrophages from rat lung tissue infected with Mycobacterium tuberculosis.

\section{ANALYSIS OF CD133 AND CXCR4 EXSPRESSION IN OSTEOSARCOMA WITH METASTASIS}

\author{
Nunik Hapsari Susilowati, Sjahjenny Mustokoweni \\ Pathology Department, Dr. Soetomo Hospital, School of \\ Medicine Airlangga University, Surabaya, Indonesia
}

Background: Osteosarcoma is associated with poor prognosis due to its high incidence of metastasis and chemoresistance and in osteosarcoma it can be identified by using tumor marker CD 133. In osteosarcoma it has high growth potential and contributes to the incidence of metastases. Several studies showed that CXCR4 as chemokine receptor in GPCR gene family, has been proven to play an essential role in the metastasis of cancer stem cell.

Objective: To analyze the correlation of CD 133 and CXCR4 expression with incidence of metastasis in osteosarcoma.

Methods: An analytic observational design with cross sectional approach. Study sample was patients with osteosarcoma that histopathologically diagnosed at the Pathology Laboratory Dr. Soetomo Hospital Surabaya during 2007-2013. Incidence of metastasis was collected from medical record. Immunohistochemical examination using rabbit polyclonal antibody CD 133 and CXCR4 (Boster, Fremont, USA). Expression of CD 133 and CXCR4 were assessed based on the number of tumor cells that showed imunoreactivity semiquantitatively. Correlations were analyzed using Chi square test and Spearman correlation test with significance on $p<0.05$.

Result: There were 9 cases of 24 osteosarcoma with metastasis. 7 of $9(77.8 \%)$ showed moderate to strong expression of CD 133 and CXCR4. There was correlation between CD 133 and CXCR4 expression with the occurrence of metastases ( $p=0.035$ and $p=0.035$ ) and there was a positive correlation between the expression of CD133 and CXCR4 $(p=0.000)$. 